\title{
Adverse pregnancy outcomes in adolescents and young women with systemic lupus erythematosus: a national estimate
}

Nicole Ling ${ }^{*}$ (D), Erica Lawson and Emily von Scheven

\begin{abstract}
Background: Pregnant women with systemic lupus erythematosus (SLE) have increased risk of adverse outcomes including disease flare, spontaneous abortion, preeclampsia/eclampsia, premature birth and maternal death. However, pregnancy outcomes among adolescents and young women with SLE have not been well-explored. Our objective was to compare risk of adverse pregnancy outcomes in adolescents and young women with SLE to risk among peers without SLE.

Methods: We studied the 2000-2011 Nationwide Inpatient Sample (NIS) of the Healthcare Cost and Utilization Project (HCUP) to estimate the prevalence of adverse pregnancy outcomes in women with SLE aged $\leq 21$ years at time of delivery. Outcomes were compared to peers without SLE by using multivariate logistic regression to calculate odds ratios and risk differences. Additionally, differences in length of stay and total charges per hospitalization were described.

Results: There were 8,791,391 unique pregnancies, of which 4002 occurred in young women with SLE. After adjustment for age, race, insurance type and quartile of median income based on patient ZIP code individuals with SLE had increased odds of pre-eclampsia/eclampsia (OR 3.2, 95\% Cl 2.3-4.6), maternal death (OR 80, 95\% Cl 10-604), preterm birth (OR 2.7, $95 \% \mathrm{Cl} 2-3.7$ ), spontaneous abortion (OR 5.1, 95\% Cl 2.8-9.6), and induced abortion (OR 30, 95\% Cl 14-63). The increase in risk among women with SLE was greatest for preterm birth (RD 11\%, 95\% Cl 6-16), pre-eclampsia/eclampsia (RD 9\%, 95\% Cl 5-13), and spontaneous abortion (RD 4\%, 95\% Cl 0.9-6). Risk difference for induced abortion was $2 \%$ with 95\% Cl 0.6-4, while the difference in risk for maternal death did not reach statistical significance (RD $0.4,95 \% \mathrm{Cl}-0.4-1$ ).

Conclusions: Adolescents and young women with SLE experience increased risk of adverse, pregnancy-specific outcomes as compared to their peers, including pre-eclampsia/eclampsia, maternal death, preterm birth, spontaneous abortion, and induced abortion. Additionally, length of stay and total charges for hospitalization are increased.
\end{abstract}

Keywords: Pregnancy, Systemic lupus erythematosus, Outcomes, Adolescent

\footnotetext{
* Correspondence: nicole.ling@ucsf.edu

University of California San Francisco, 550 16th Street, 5th Floor, Box 0632,

San Francisco, CA 94143, USA
}

(c) The Author(s). 2018 Open Access This article is distributed under the terms of the Creative Commons Attribution 4.0 International License (http://creativecommons.org/licenses/by/4.0/), which permits unrestricted use, distribution, and reproduction in any medium, provided you give appropriate credit to the original author(s) and the source, provide a link to the Creative Commons license, and indicate if changes were made. The Creative Commons Public Domain Dedication waiver (http://creativecommons.org/publicdomain/zero/1.0/) applies to the data made available in this article, unless otherwise stated. 


\section{Background}

Systemic Lupus Erythematosus (SLE) is a complex autoimmune condition that affects adolescents and young women of childbearing age. When women with SLE become pregnant, numerous manifestations of SLE and its associated treatment can affect both maternal and fetal outcomes. Prevalence of adverse pregnancy outcomes in the SLE adult population have been well documented, including estimates from several single-center cohort studies from across the world [1-10]. Comparative studies using national registries have reported increased risk of preterm labor, fetal growth restriction, hypertensive disorders including pre-eclampsia and eclampsia, maternal death, and fetal death in SLE [11-13]. Women with SLE are also at increased risk of infectious, hematologic and thrombotic maternal complications. Risk factors associated with poor pregnancy outcomes among women with SLE include active disease, renal involvement, presence of anti-phospholipid antibodies, use of high doses of glucocorticoids, and fewer years of education [14-18].

Young women and adolescents are at increased risk of unintended pregnancy as compared to older women [19]. Sexual behavior of adolescents with chronic health conditions has been shown to be no different than that of their peers without chronic conditions, putting young women with SLE at the same risk of unintended pregnancy [20]. Furthermore, there is increased incidence of renal involvement among adolescents with SLE as compared to adults with SLE, which may put adolescents at greater risk of poor obstetric outcomes if they become pregnant [21].

In spite of these increased risks, pregnancy incidence and outcomes among adolescents and young women with SLE remain understudied. In the last ten years, only one study has attempted to capture pregnancy risk in this population [22]. Prevalence of maternal and fetal outcomes was described in a multi-center, Brazilian cohort of 24 pregnancies in 23 patients with pediatric-onset SLE, aged 14-21.7 years. Using multivariate modeling, inadvertent intravenous cyclophosphamide use at the start of pregnancy was the only independent risk factor for fetal loss. However, the sample size for the study was small and comparative measures of risk between those with and without SLE were not explored.

In the current study, we used a national dataset to estimate risk of adverse, pregnancy-specific outcomes among adolescents and young women with SLE, focusing on the age range typically cared for by pediatric rheumatologists.

\section{Methods}

\section{Data source}

We studied the 2000-2011 Nationwide Inpatient Sample (NIS), part of the Agency for Healthcare Research and Quality's Healthcare Cost and Utilization Project (HCUP) [23]. The NIS is the largest all-payer, inpatient care database in the United States, containing demographic and clinical information obtained from hospital discharge abstracts. Available yearly since 1988, each year of the NIS contains data on approximately 8 million hospital stays from about 1000 hospitals, sampled to approximate a $20 \%$ stratified sample of U.S. community hospitals. Hospitals are divided into strata based on U.S. region, urban/rural location, teaching status, bed size and ownership. Sampling probabilities are proportional to the number of hospitals in each stratum. No unique patient identifiers are contained in the NIS, as the unit of analysis is individual hospitalization, rather than patient. Information available for each hospitalization includes general hospital characteristics as well as patient information such as age, gender, race, quartile of median income based on patient ZIP code, and discharge diagnoses/procedure codes from the International Classification of Diseases, Ninth Revision, Clinical Modification (ICD-9-CM). Discharge diagnoses include one primary and up to 24 secondary discharge diagnoses as well as up to 15 procedural codes.

\section{Study population}

We identified unique pregnancies among individuals with and without SLE age 14-21 years. We chose to include individuals up to age 21 because previous survey data demonstrates that nearly half of pediatric rheumatologists follow patients until age 21 or older [24]. Hospitalizations of patients with SLE were identified with ICD-9 code 710.0. The youngest, pregnant patient with SLE was 14 years of age at time of delivery, so analysis was restricted to hospitalizations of pregnant women aged 14 or older. Hospitalizations of pregnant women aged 14-21 were included. Unique pregnancies were identified for analysis by including only those hospitalizations in which ICD-9 diagnosis or procedural codes specified delivery, live born, spontaneous or induced abortion, ectopic pregnancy or intrauterine death. ICD-9 codes for these conditions were identified using HCUP Clinical Classifications Software (CCS) and incorporated for inclusion/exclusion using ICD9x for STATA 13.0 software [25-27].

\section{Measures}

The primary outcome was pregnancy-specific adverse maternal or fetal outcome. Maternal outcomes included pre-eclampsia/eclampsia and death. Fetal outcomes included preterm birth (early onset of delivery, immaturity or birth before 37 weeks gestation), spontaneous abortion (unspecified abortion or intrauterine death), induced abortion, and ectopic pregnancy. Data on other fetal outcomes such as weight, growth and congenital anomalies were not reliably available from maternal records, and therefore were not included. ICD-9 codes for each of the above variables are available in tables provided in the 
Additional file 1. Additionally, length of stay and total charges per hospital stay were included as secondary outcome variables. Predictor variables included age, race, socioeconomic status (SES) and insurance statues. Race was categorized as White, Black, Hispanic, Asian or Pacific Islander, Native American, or Other. SES was categorized according to quartile of median household income based on patient ZIP code. Insurance status was defined as primary expected payor.

\section{Statistical analysis}

Sample weights were applied to account for stratified sampling design and multiple years of data, generating nationally representative estimates. Survey data commands were used for all descriptive and inferential analyses. Unadjusted linear regression was used to compare age, length of stay and total charges in those with and without SLE. Chi squared analyses were used to compare weighted cell frequencies and their proportions. Adjusted logistic regression was used to compute odds ratios and 95\% confidence intervals for the primary outcome of pregnancy-specific adverse maternal and fetal outcomes among women aged 14-21 with and without SLE. Marginal estimates were calculated to obtain risk difference for these outcomes. Both the odds ratio (OR) and risk difference (RD) calculations were performed after adjusting for age, race, SES and insurance. There was missing data for some observations of race, as some hospitals and HCUP State Partners do not supply this information. Therefore, to account for missing data, inverse probability weights were generated using logistic regression to model for race "missingness" and then applied prior to calculation of adjusted risk estimates [23, 28]. The logistic regression model for race "missingness" included patient age, number of chronic diseases, NIS stratum, quartile of median income based on patient ZIP code, SLE status, and all maternal pregnancy outcome variables. Unstabilized weights were used due to the lack of variation in weights. Descriptive statistics were used to compare secondary outcomes, length of stay and total charges per hospital stay, among women with and without SLE.

All statistical analyses were performed using STATA software, version 13.0 (StataCorp, College Station, TX.)

\section{Results}

\section{Obstetric hospitalizations}

From 2000 to 2011, there were 8,791,391 hospitalizations for unique pregnancies among young women age 14-21 (Table 1). Among these hospitalizations, 4002 included a discharge diagnosis of SLE. The mean age was 19.4 years in the SLE group and 19.0 in the non-SLE group $(p<0.001)$. Distribution of race across SLE and non-SLE differed $(\mathrm{p}<0.001)$. The non-SLE group included more Caucasians (43\% vs. 35\%), while the SLE group included more African Americans (34\% vs. $21 \%$ ). There was no difference in
Table 1 Demographics of pregnant, hospitalized, young women with and without SLE, age 14-21

\begin{tabular}{|c|c|c|c|}
\hline \multirow[t]{2}{*}{ Demographic } & $\begin{array}{l}\text { SLE } \\
(n=4002)\end{array}$ & $\begin{array}{l}\text { Non-SLE } \\
(n=8,787,389)\end{array}$ & \multirow[t]{2}{*}{$P$} \\
\hline & \multicolumn{2}{|c|}{$N(\%)$ or Mean $( \pm S D)$} & \\
\hline Age at delivery, years & $19.4 \pm 0.05$ & $19.0 \pm 0.01$ & $<0.001$ \\
\hline Race $(n=6,646,192)$ & & & $<0.001$ \\
\hline White & $1107(35)$ & $2,837,585(43)$ & \\
\hline Black & $1070(34)$ & $1,402,261(21)$ & \\
\hline Hispanic & $828(26)$ & $1,937,211(29)$ & \\
\hline Asian or Pacific Islander & $43(1.3)$ & $114,299(1.7)$ & \\
\hline Native American & $39(1.2)$ & $62,090(0.93)$ & \\
\hline Other & $91(2.9)$ & $289,569(4.4)$ & \\
\hline $\begin{array}{l}\text { Socioeconomic Status }{ }^{a} \\
(n=8,622,189)\end{array}$ & & & 0.42 \\
\hline 1 & $1273(32)$ & $2,738,962(32)$ & \\
\hline 2 & $1134(29)$ & $2,581,298(30)$ & \\
\hline 3 & $853(22)$ & $1,995,447(23)$ & \\
\hline 4 & $665(17)$ & $1,302,558(15)$ & \\
\hline Insurance Type $(n=8,774,388)$ & & & $<0.001$ \\
\hline Medicare & $43(1.1)$ & $29,987(0.3)$ & \\
\hline Medicaid & $2666(67)$ & $6,082,943(69)$ & \\
\hline Private, including $\mathrm{HMO}$ & $1048(26)$ & $2,070,621(24)$ & \\
\hline Self-pay & $89(2.2)$ & $329,328(3.8)$ & \\
\hline No charge & $9(0.2)$ & $22,538(0.3)$ & \\
\hline Other & $138(3.4)$ & $234,979(2.7)$ & \\
\hline
\end{tabular}

${ }^{\mathrm{a}}$ As represented by median household income quartiles for patient's ZIP code: $1=$ lowest income, $4=$ highest income

socioeconomic status between SLE and non-SLE groups, as represented by median household income quartile based on patient zip code. A difference in insurance type between the two groups was detected. Those with SLE had a slightly increased prevalence of private insurance ( $26 \%$ vs. $24 \%$ ) and a slightly lower prevalence of Medicaid (67\% vs. $69 \%)$ and self-pay (2.2\% vs. $3.8 \%)$ when compared to those with SLE $(p<0.001)$.

\section{Adverse pregnancy outcomes}

Prevalence of pre-eclampsia and eclampsia, maternal death, preterm birth, spontaneous abortion, and induced abortion were increased in those with SLE compared to those without SLE in unadjusted analysis (Table 2). After adjusting for age, race, insurance type, and quartile of median household income based on patient ZIP code, SLE was associated with increased odds of pre-eclampsia/ eclampsia (OR 3.2, 95\% CI 2.3-4.6), maternal death (OR $80,95 \%$ CI 10-604), preterm birth (OR 2.7, 95\% CI 2-3.7), spontaneous abortion (OR 5.1, 95\% CI 2.8-9.6), and induced abortion (OR 30, 95\% CI 14-63). Ectopic pregnancies were 
Table 2 Adverse pregnancy outcomes of young women age 14-21 with and without SLE

\begin{tabular}{|c|c|c|c|c|}
\hline \multirow[t]{2}{*}{ Outcome } & $\operatorname{SLE}(n=4002)$ & Non-SLE $(n=8,787,389)$ & $\mathrm{OR}(95 \% \mathrm{Cl})^{\mathrm{a}}$ & Risk Difference \% $(95 \% \mathrm{Cl})^{\mathrm{a}}$ \\
\hline & \multicolumn{4}{|l|}{ N (\%) } \\
\hline \multicolumn{5}{|l|}{ Maternal } \\
\hline Pre-eclampsia or eclampsia & $636(16)$ & $401,549(4.6)$ & $3.2(2.3-4.6)$ & $9(5-13)$ \\
\hline Death & $15(0.38)$ & $465(0.005)$ & $80(10-604)$ & $0.4(-0.4-1)$ \\
\hline \multicolumn{5}{|l|}{ Fetal } \\
\hline Preterm Birth & $818(20)$ & $707,921(8.1)$ & $2.7(2-3.7)$ & $11(6-16)$ \\
\hline Spontaneous abortion or Intrauterine death & $221(5.5)$ & $100,130(1.1)$ & $5.1(2.8-9.6)$ & $4(0.9-6)$ \\
\hline Induced abortion & $87(2.2)$ & $11,175(0.13)$ & $30(14-63)$ & $2(0.6-4)$ \\
\hline Ectopic pregnancy & $\leq 10(\leq 0.24)^{b}$ & $29,095(0.3)$ & $0.8(0.12-6)$ & $-0.1(-0.7-0.6)$ \\
\hline
\end{tabular}

${ }^{a}$ Adjusted for age, race, insurance type, and quartile of median income based on patient ZIP code

${ }^{b}$ Unable to report cell sizes less than or equal to 10 , per HCUP data use regulations

rare in this population and differences across those with and without SLE did not reach statistical significance.

The increase in risk among women with SLE was greatest for preterm birth (RD 11\%, 95\% CI 6-16), preeclampsia/eclampsia (RD 9\%, 95\% CI 5-13), and spontaneous abortion (RD 4\%, 95\% CI 0.9-6). Risk difference for induced abortion was $2 \%$ with $95 \%$ CI $0.6-4$, while the difference in risk for maternal death did not reach statistical significance (RD 0.4, 95\% CI -0.4-1).

\section{Healthcare utilization}

Length of stay and total charges per hospitalization were compared between the SLE and non-SLE group. Median length of stay for those with SLE was 3 days (interquartile range (IQR) 2-4 days) compared to 2 days (IQR 1-3 days) in those without SLE $(p<0.001)$. Median total charges for hospitalization for those with SLE was \$11,146 (IQR \$6973-\$19,187) compared to \$7198 (IQR \$4824-\$11,172) for those without SLE $(\mathrm{p}<0.001)$.

\section{Discussion}

This study demonstrated an increased risk of adverse pregnancy-related outcomes among adolescents and young women with SLE as compared to their peers without SLE, utilizing a large nationwide dataset. Compared to those without SLE, pregnant young women with SLE were at increased risk for pre-eclampsia/eclampsia, maternal death, spontaneous and induced abortion, and preterm birth. Prior studies have not focused on the population typically cared for by pediatric rheumatologists, in spite of the fact that pregnancy occurs frequently among adolescents, including those with SLE $[2,3]$.

The increased risk of adverse, pregnancy-specific outcomes among adolescents and young women with SLE is consistent with previous findings in the literature among adult women with SLE. Studies using national registries have reported increased risk of preterm labor (OR 2.4-4.0), fetal growth restriction (OR 2.6-5.0), hypertensive disorders including pre-eclampsia and eclampsia (OR 3-4.4), maternal death (OR 17.8), and fetal death (OR 2.4-7) [11-13] Estimates of risk of maternal death in those aged 21 or less are slightly higher than previously published work looking at adult populations. However, it should be noted that this outcome was still a rare occurrence and our estimates included a wide confidence interval. Risk differences were calculated to further explore the risk of maternal death. Risk difference calculations take into account the prevalence of each outcome in the study population. Though maternal death and induced abortion had the highest odds ratios, these outcomes had the lowest differences in risk, and the risk difference for maternal death was not significant. The findings of increased adverse pregnancy outcomes in those with SLE are further strengthened by detected differences in median length of stay and total charges for hospitalization.

Because of the sampling methodology used in the study design of the NIS database, our results are generalizable to hospitals included in the sampling frame. In 2011, the NIS captured over $97 \%$ of the U.S. population hospital discharges from 46 states. Therefore, our results are generalizable to nearly the entire U.S. population.

Use of this large, nationally-representative dataset has inherent limitations. First, classification of SLE and adverse maternal pregnancy outcomes were based on ICD-9 discharge diagnosis codes. These discharge diagnosis codes are subject to coding error and misclassification. We were unable to validate the sensitivity or specificity of coding in this study, however, previous research has estimated the positive predictive value (PPV) of ICD-9 billing codes for SLE and SLE nephritis in Medicaid patients. These authors studied several algorithms to attempt to capture patients with SLE and SLE nephritis. Their algorithms included three or more ICD-9 codes for SLE (710.0) and either/both of the following: three or some renal ICD9 codes and 3 or more nephrology visits. Using these algorithms combined with chart review, PPV for SLE ranged from 89 to 92\%) [29]. As mentioned previously, there are no unique patient identifiers in the NIS database, therefore inclusion of repeated ICD-9 codes to define SLE in this 
study was not possible. Another study based in Canada calculated sensitivity, specificity, PPV and negative predictive values (NPV) of ICD-9 codes. They reported a sensitivity of 89.8, specificity of 98.7, PPV of 99.9, and NPV of 0.64 for detecting rheumatic disease with ICD-9 coding in their database [30]. Of note, misclassification of SLE in this study would decrease our ability to detect differences between the two groups.

Second, we are limited by the amount of information provided by hospital discharge abstracts. We were unable to examine the impact of SLE related covariates such as disease duration, damage, and activity. Furthermore, effects of medications and behavioral characteristics of patients (adherence, access to prenatal care) on adverse pregnancy-specific outcomes could not be explored due to lack of medical information in the dataset. Third, we were unable to link maternal hospital discharge records to corresponding fetal hospital discharge records, limiting our ability to explore fetal-specific outcomes, including length of stay and total charges for hospitalization. Finally, the NIS contains only data from hospitalizations. Deliveries and abortions that did not occur in the hospital setting are not accounted for in this analysis, which may bias estimates if those with SLE are more likely to present to the hospital for delivery and abortion as compared to women without SLE. In spite of these limitations, the size of this dataset enabled us to estimate risk for rare events of great interest to patients and clinicians, specifically adverse pregnancy-specific outcomes among young women with SLE, that might otherwise not be possible.

\section{Conclusions}

These results are the first to quantify risk of adverse, pregnancy-specific outcomes in adolescents and young women with SLE on a national scale. The increase in risk among women with SLE was greatest for preterm birth, pre-eclampsia/eclampsia, and spontaneous abortion. This work provides practitioners with tangible estimates of risk for young women and adolescents that can be leveraged when discussing reproductive health and when counseling adolescents who become pregnant. Further studies to identify specific disease and treatment related risk factors in the adolescent and young women population are needed.

\section{Additional file}

Additional file 1: Table S1a. ICD9 diagnosis and procedure codes used to identify hospitalizations for unique pregnancies. Table S1b. ICD9 diagnosis codes used to identify pregnancy outcomes. (PDF $50 \mathrm{~kb}$ )

\section{Abbreviations}

Cl: confidence interval; HCUP: Healthcare Cost and Utilization Project: ICD-9-CM: International Classification of Diseases, Ninth Revision, Clinical Modification; NIS: Nationwide Inpatient Sample; NPV: negative predictive value; OR: odds ratio; PPV: positive predictive value; RD: risk difference;
SLE: systemic lupus erythematosus; U.S.: United States; ZIP code: zone improvement plan code

Funding

NIAMS 2T32AR007304-36, Arthritis Foundation PDF 6111

\section{Availability of data and materials}

The data that support the findings of this study are available from Healthcare Cost and Utilization Project but restrictions apply to the availability of these data, which were used under license for the current study, and so are not publicly available. Data are however available from the authors upon reasonable request and with permission of Healthcare Cost and Utilization Project.

\section{Author's contributions}

$\mathrm{NL}, \mathrm{EL}$ and EVS contributed to the conception and study design. NL aquired the data and performed the analysis, with interpretation of the data by all authors. All authors have had significant input in the drafting and revision of the manuscript and have approved the final manuscript.

Ethics approval and consent to participate

The University of California, San Francisco Committee on Human Research did not require review for research performed on de-identified data.

\section{Competing interests}

The authors declare that they have no competing interests.

\section{Publisher's Note}

Springer Nature remains neutral with regard to jurisdictional claims in published maps and institutional affiliations.

Received: 16 January 2018 Accepted: 4 April 2018

Published online: 16 April 2018

\section{References}

1. Madazli R, Bulut B, Erenel H, Gezer A, Guralp O. Systemic lupus erythematosus and pregnancy. Journal of obstetrics and gynaecology : the journal of the Institute of Obstetrics and Gynaecology. 2010;30:17-20.

2. Cavallasca JA, Laborde HA, Ruda-Vega H, Nasswetter GG. Maternal and fetal outcomes of 72 pregnancies in argentine patients with systemic lupus erythematosus (SLE). Clin Rheumatol. 2008;27:41-6.

3. Carvalheiras G, Vita P, Marta S, et al. Pregnancy and systemic lupus erythematosus: review of clinical features and outcome of 51 pregnancies at a single institution. Clinical reviews in allergy \& immunology. 2010;38:302-6.

4. Aggarwal N, Raveendran A, Suri V, Chopra S, Sikka P, Sharma A. Pregnancy outcome in systemic lupus erythematosus: Asia's largest single Centre study. Arch Gynecol Obstet. 2011:284:281-5

5. Ambrosio P, Lermann R, Cordeiro A, Borges A, Nogueira I, Serrano F. Lupus and pregnancy-15 years of experience in a tertiary center. Clinical reviews in allergy \& immunology. 2010;38:77-81.

6. Teh CL, Wong JS, Ngeh NK, Loh WL. Systemic lupus erythematosus pregnancies: the Sarawak experience and review of lupus pregnancies in Asia. Rheumatol Int. 2011;31:1153-7.

7. Whitelaw DA, Hall D, Kotze T. Pregnancy in systemic lupus erythematosus: a retrospective study from a developing community. Clin Rheumatol. 2008;27: 577-80

8. Wong $\mathrm{CH}$, Chen TL, Lee CS, Lin CJ, Chen CP. Outcome of pregnancy in patients with systemic lupus erythematosus. Taiwanese journal of obstetrics \& gynecology. 2006;45:120-3

9. Ideguchi $\mathrm{H}$, Ohno S, Uehara $\mathrm{T}$, Ishigatsubo $\mathrm{Y}$. Pregnancy outcomes in Japanese patients with SLE: retrospective review of 55 pregnancies at a university hospital. Clinical reviews in allergy \& immunology. 2013;44:57-64.

10. Chandran V, Aggarwal A, Misra R. Active disease during pregnancy is associated with poor foetal outcome in Indian patients with systemic lupus erythematosus. Rheumatol Int. 2005:26:152-6.

11. Clowse ME, Jamison M, Myers $E_{1}$ James $A H$. A national study of the complications of lupus in pregnancy. Am J Obstet Gynecol. 2008;199(127):e1-6.

12. Chakravarty EF, Nelson L, Krishnan E. Obstetric hospitalizations in the United States for women with systemic lupus erythematosus and rheumatoid arthritis. Arthritis Rheum. 2006;54:899-907. 
13. Wallenius M, Salvesen KA, Daltveit AK, Skomsvoll JF. Systemic lupus erythematosus and outcomes in first and subsequent births based on data from a national birth registry. Arthritis care \& research. 2014;66:1718-24.

14. Andrade R, Sanchez ML, Alarcon GS, et al. Adverse pregnancy outcomes in women with systemic lupus erythematosus from a multiethnic US cohort: LUMINA (LVI) [corrected]. Clin Exp Rheumatol. 2008;26:268-74.

15. Wagner SJ, Craici I, Reed D, et al. Maternal and foetal outcomes in pregnant patients with active lupus nephritis. Lupus. 2009;18:342-7.

16. Ko HS, Ahn HY, Jang DG, et al. Pregnancy outcomes and appropriate timing of pregnancy in 183 pregnancies in Korean patients with SLE. Int J Med Sci. 2011;8:577-83

17. Madazli R, Yuksel MA, Oncul M, Imamoglu M, Yilmaz H. Obstetric outcomes and prognostic factors of lupus pregnancies. Arch Gynecol Obstet. 2014;289:49-53.

18. Chakravarty EF, Colon I, Langen ES, et al. Factors that predict prematurity and preeclampsia in pregnancies that are complicated by systemic lupus erythematosus. Am J Obstet Gynecol. 2005;192:1897-904.

19. Finer $L B$, Zolna MR. Shifts in intended and unintended pregnancies in the United States, 2001-2008. Am J Public Health. 2014;104(Suppl 1):S43-8.

20. Suris JC, Resnick MD, Cassuto N, Blum RW. Sexual behavior of adolescents with chronic disease and disability. The Journal of adolescent health : official publication of the Society for Adolescent Medicine. 1996;19:124-31.

21. Brunner HI, Gladman DD, Ibanez D, Urowitz MD, Silverman ED. Difference in disease features between childhood-onset and adult-onset systemic lupus erythematosus. Arthritis Rheum. 2008;58:556-62.

22. Silva CA, Hilario MO, Febronio MV, et al. Pregnancy outcome in juvenile systemic lupus erythematosus: a Brazilian multicenter cohort study. J Rheumatol. 2008;35:1414-8.

23. HCUP Nationwide Inpatient Sample (NIS). Healthcare Cost and Utilization Project (HCUP). 2000-2011. Agency for Healthcare Research and Quality, Rockville, MD. at http://www.hcup-us.ahrq.gov/nisoverview.sp.)

24. Chira P, Ronis T, Ardoin S, White P. Transitioning youth with rheumatic conditions: perspectives of pediatric rheumatology providers in the United States and Canada. J Rheumatol. 2014;41:768-79.

25. Rising B. icd9x: A program for marking observations on ICD-9 inclusion/ exclusion.This program makes it simple to implement inclusion/exclusion criteria when using multiple icd9 codes to classify disease.

26. HCUP Clinical Classifications Software (CCS) for ICD-9-CM. Healthcare Cost and Utilization Project (HCUP). 2006-2009. Agency for Healthcare Research and Quality, Rockville, MD. 2014, at http://www.hcup-us.ahrq.gov/toolssoftware/ccs/ccs.jsp.)

27. StataCorp. Stata statistical software: release 13. College Station. TX: StataCorp LP; 2013.

28. Foreman EK. Survey sampling principles. New York: CRC Press; 1991.

29. Chibnik LB, Massarotti EM, Costenbader KH. Identification and validation of lupus nephritis cases using administrative data. Lupus. 2010;19:741-3.

30. Quan H, Li B, Duncan Saunders L, et al. Assessing validity of ICD-9-CM and ICD-10 administrative data in recording clinical conditions in a unique dually coded database. Health Serv Res. 2008;43:1424-41.

\section{Ready to submit your research? Choose BMC and benefit from:}

- fast, convenient online submission

- thorough peer review by experienced researchers in your field

- rapid publication on acceptance

- support for research data, including large and complex data types

- gold Open Access which fosters wider collaboration and increased citations - maximum visibility for your research: over $100 \mathrm{M}$ website views per year

At BMC, research is always in progress.

Learn more biomedcentral.com/submissions 